\title{
Human-AI Collaboration in Healthcare: A Review and Research Agenda
}

\author{
Yi Lai \\ Department of Information Systems \\ and Analytics, National University \\ of Singapore \\ laiyi@u.nus.edu
}

\author{
Atreyi Kankanhalli \\ Department of Information Systems \\ and Analytics, National University \\ of Singapore \\ atreyi@comp.nus.edu.sg
}

\author{
Desmond C. Ong \\ Department of Information Systems \\ and Analytics, National University \\ of Singapore \\ dco@comp.nus.edu.sg
}

\begin{abstract}
Advances in Artificial Intelligence (AI) have led to the rise of human-AI collaboration. In healthcare, such collaboration could mitigate the shortage of qualified healthcare workers, assist overworked medical professionals, and improve the quality of healthcare. However, many challenges remain, such as investigating biases in clinical decision-making, the lack of trust in AI and adoption issues. While there is a growing number of studies on the topic, they are in disparate fields, and we lack a summary understanding of this research. To address this issue, this study conducts a literature review to examine prior research, identify gaps, and propose future research directions. Our findings indicate that there are limited studies about the evolving and interactive collaboration process in healthcare, the complementarity of humans and AI, the adoption and perception of AI, and the longterm impact on individuals and healthcare organizations. Additionally, more theory-driven research is needed to inform the design, implementation, and use of collaborative AI for healthcare and to realize its benefits.
\end{abstract}

\section{Introduction}

Artificial intelligence (AI) refers to the attempt to reproduce humans' cognitive abilities using artificial, computer systems [27]. AI systems can now learn from data, identify patterns and make decisions. After years of advances in AI techniques, especially with the emergence of deep learning algorithms, AI has finally left the realm of science fiction and become commercially important. For example, autonomous driving is a key application of AI, with the projected value of the global autonomous vehicle market expected to reach $\$ 557$ billion by 2026 [2].

Thus, AI along with other computing technologies is transforming the way in which businesses and industries operate. Businesses are seeing a number of jobs being replaced entirely by AI e.g., telemarketers, and receptionists, but they are also finding means to use AI to augment existing human capital. According to Deloitte's Global Human Capital Trends survey [6], $60 \%$ of respondents stated that their organization was using AI to assist rather than to replace workers.
Furthermore, organizations reported using AI to assist workers mainly for consistency and quality improvements, as well as productivity enhancement. This shows evidence of the emerging phenomenon of human-AI collaboration, which is suggested to improve performance in multiple ways, as compared to using AI as a tool [28]. We define collaboration as an evolving, interactive process whereby two or more parties actively and reciprocally engage in joint activities aimed at achieving one or more shared goals [3]. Human-AI collaboration then refers to the collaboration between single or multiple humans and AI systems. In contrast to the situation where AI systems were mainly automating routine human tasks in the past, human-AI collaboration implies that AI systems work jointly with humans like teammates or partners to solve problems. For example, consider a clinical decision support system (CDSS) for diagnosing the stage of cancer collaborating with the physician to complete the diagnosis.

Indeed, healthcare is a critical context for humanAI collaboration. The World Health Organization in its 2019 World Health Report, highlights a persistent global shortage of physicians, with an average of only 15 doctors per 10,000 people [29]. Furthermore, medical professionals are routinely overworked - and even more so during pandemics, which leads to decreases in healthcare quality and potential lifethreatening human errors [25]. Given such severe manpower shortages, human-AI collaboration that augments the work of healthcare professionals could reduce their workload and improve the quality of service, which in turn can positively impact many health-related outcomes. For example, empirical studies have shown that CDSS can improve healthcare professionals' efficiency [24] and effectiveness [14]. Collaborations of humans and AI systems are seen to achieve superior performance using collective intelligence [28]. For example, Tschandl and colleagues found that AI-supported clinical decision-making improved skin cancer diagnostic accuracy over that of either AI or physicians alone [57].

Other than the challenges facing healthcare professionals, for patients and their families, it is reported that as little as $3 \%$ of the U.S. population can afford even part-time care support from a human caregiver [20]. At the same time, there is an increasing 
demand for caregivers, with the widespread prevalence of chronic diseases and ageing-related ailments. This suggests a value proposition for interactive caregiver robots or other AI systems that can collaborate with patients to improve healthcare. These patients may then be able to enjoy more engagement and better quality of care at reduced costs.

In order to identify the current state of research in human-AI collaboration in healthcare, we conducted a literature review to investigate how researchers across multiple disciplines studied the design and implementation of AI systems for collaboration with humans in healthcare, the adoption and use of such systems, and the evaluation of outcomes of such collaboration. We aimed to identify gaps in understanding and propose directions to guide future IS research. This paper is structured as follows. First, we introduce related work and research methodology. We then categorize and discuss the distribution of papers along various aspects. Subsequently, we synthesize major themes from our review and identify gaps in understanding. Finally, we conclude the paper by proposing future research directions.

\section{Related work}

Although the capabilities of modern AI have been largely improved with the development of big data and deep learning techniques, there are still multiple challenges to achieving the benefits of human-AI collaboration in healthcare. First, researchers have pointed out the perils of using biased AI systems, e.g., diagnostic systems using datasets that are imbalanced with respect to race or other demographics [19]. Biased AI systems can diminish rather than augment human intelligence in collaborative decision-making. Second, many machine learning methods, especially deep learning models, lack interpretability and transparency. They are typically "black box" models that are unable to give a rationale or explanations for their decisions. This may impair the trust between healthcare users and the AI system, which in turn hinders their collaboration [50]. Third, there are still critical issues with healthcare users' adoption of AI technology, which implicates human-AI collaboration. In a poll conducted by HIMSS Analytics, over a third of healthcare professionals expressed apprehension about adopting AI due to concerns about the alignment of the AI systems goals with theirs, and the perceived immaturity of the technology [10]. Fourth, there are multiple challenges related to the behavioral and social aspects of human-AI collaboration in healthcare, which also impact their adoption. Other than the lack of trust in AI [50] [31], humans and AI systems are often unable to infer each other's goals and intentions [11], which is necessary to resolve conflicts that may occur. In healthcare, there are also concerns about who is accountable for the outcomes in a human-AI collaboration [21], e.g., a treatment decision that results in a patient's death. Psychologically, issues of autonomy and control also surface when healthcare professionals are expected to consider AI systems as teammates rather than tools [23]. In the medical profession particularly, clinical autonomy has remained the defining characteristic of power and status of healthcare professionals, which would be difficult for them to relinquish [1]. Finally, there are organizational challenges around human-AI collaboration in healthcare. For instance, healthcare organizations (e.g., hospitals) are not necessarily convinced about the performance impacts of human-AI collaboration, which hinders their investments in collaborative AI systems. According to a survey conducted by Olive AI in 2019, just $23 \%$ of hospital executives said they were seeking to invest in AI and robotic process automation (RPA) solutions today [17]. Additionally, organizations are often unaware of the changes needed in workflows and the required skills for professionals to collaborate with AI systems [23].

In sum, human-AI collaboration in healthcare shows much promise, but also faces significant challenges, which present important opportunities for IS researchers. Technology-mediated collaboration has been a strong focus of IS research (e.g., [4]), and these insights can be used to improve human-AI collaboration in healthcare. Thus, it would be valuable to assess the prior research on this topic and identify promising directions for future research for IS scholars in this area.

In this regard, we identified five recent review papers related to the topic of human-AI collaboration. All five papers acknowledged the utility of AI applications across healthcare domains but focused on specific healthcare aspects. First, Pacis and colleagues [18] discussed various AI applications in telemedicine, proposed four trends for future applications, and identified challenges in their implementation. Focusing only on one specialization, psychotherapy, Miner and colleagues [16] outlined four approaches and dimensions of care that conversational AI will affect when integrated into mental health service delivery. Focusing on surgery and surgical data science (SDS), Vedula and Hager [26] suggested that SDS could transform passive surgical technologies into an interactive platform that can collaborate with and actively assist physicians. Motivated by the "black box" problem in AI techniques, Lai and colleagues [13] reviewed research on CDSS with respect to the role of explanations. Finally, Seeber and colleagues [23] surveyed 65 collaboration researchers and developed a research agenda for team collaboration with AI, comprising three design areas, i.e., machine artifact design, collaboration design, and institution design. 
However, this was for team collaboration in general, and not specific to healthcare

Thus, we did not find a review addressing the research on human-AI collaboration in healthcare in general, which is our objective. This is highly relevant to IS researchers, including scholars who study collaboration, AI, and healthcare, and who may not focus on a particular healthcare specialization.

\section{Research methodology}

This study followed the prescribed steps to conduct a systematic literature review [12]. To include papers published across multiple relevant disciplines, such as computer science, information systems, health informatics, and medicine, we searched five major databases, i.e., INFORMS PubsOnLine, AIS eLibrary, PubMed, Scopus and ACM Digital Library. To cover the major IS journals, we also separately searched the Senior Scholars' Basket of Eight IS Journals, i.e., European Journal of Information Systems, Information Systems Journal, Information Systems Research, Journal of Association for Information Systems, Journal of Information Technology, Journal of Management Information Systems, Journal of Strategic Information Systems, and MIS Quarterly. We considered both peerreviewed conference and journal papers.

Particularly, in the last decade, the availability of big data and faster processing has allowed deep learning to become mainstream, enabling many breakthroughs in AI [7] [9]. Furthermore, a report from McKinsey indicated that a big data revolution in healthcare has been occurring through AI since roughly 2010 [15]. Given the rise of big data and deep learning in $\mathrm{AI}$ in the last decade, we chose the time period for our search from 2010 onwards. The search query we used was inclusive: (AI OR "artificial intelligence" OR "decision support system" OR DSS OR "machine learning" OR "deep learning" OR "neural network" OR "robot" OR "intelligent agent" OR "autonomous agent") AND (collaboration) AND ("healthcare" OR "health" OR "clinical" OR "medical").

Through a broad search using the search query on the specified databases, IS journals and time period, we identified 1019 papers as of June 2020. In the second stage, we scanned the abstracts of these papers and excluded irrelevant papers in which AI applications in healthcare were briefly mentioned as examples but were not the focus of study. This stage resulted in 633 papers. In the third stage, we scanned the full-texts of the second-stage papers and excluded irrelevant ones. These papers were mainly excluded for four reasons: 1) the word "collaboration" was only used in proper nouns, e.g., "The International Skin Imaging Collaboration Challenges"; 2) the word "collaboration" was used only to indicate the collaborators of the papers; 3 ) rather than
human-AI collaboration, the papers focused on interpersonal collaboration (e.g., patient-physician and multi-physician), multi-institutional collaboration, multi-robot collaboration and interdisciplinary collaboration; 4) the focal artifacts did not have some degree of intelligence to help human solve problems, and instead were fully-controlled by humans, such as endoscopes. After the full-text filtering, we ended up with 28 papers as relevant for our review.

\section{Findings}

In this section, we discuss the distribution of the 28 peer-reviewed conference and journal papers over time, publication outlets, diseases, clinical practices, and research methods. Last, we identify the research themes and theories employed by these 28 papers.

\subsection{Distribution over time}

As shown in Figure 1, research related to human-AI collaboration in healthcare showed an increase over time. Given the advances in AI techniques, AI systems are becoming more intelligent and working together with humans on more complex tasks than before. With the current Covid-19 pandemic, we expect more studies on this topic in the near future. There is still much potential for growth of research in this area, as the absolute number of studies is still quite small.

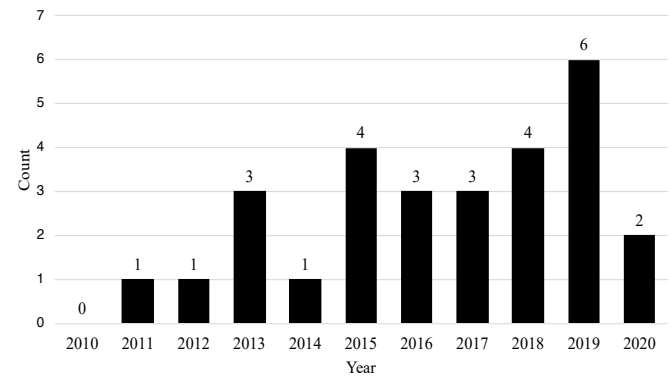

Figure 1. Number of papers across years

\subsection{Distribution over publication outlets}

The journals and conferences where the 28 papers were published are listed in Table 1. Two papers each were published in Interaction Studies and Procedia Computer Science, while the other conferences and journals had 1 paper each. As can be seen from the publication outlets (and what we expected), human-AI collaboration in healthcare is a multidisciplinary topic. These journals and conferences cover multiple fields, such as information systems, computer science, health informatics, and medicine. With the limited number of IS outlets covered, there is a largely untapped opportunity for IS journals and conferences to become more receptive to and encourage submissions in this area. 
Table 1. Publication outlets of the papers

\begin{tabular}{|l|l|l|}
\hline \multicolumn{2}{|l|}{ Conferences } & Journals \\
\hline $\begin{array}{l}\text { International Conf. on Innovations } \\
\text { in Information Technology }\end{array}$ & $\begin{array}{l}\text { International Conf on Biomedical } \\
\text { Eng. and Technology }\end{array}$ & $\begin{array}{l}\text { BMC Medical Informatics and } \\
\text { Decision Making }\end{array}$ \\
\hline $\begin{array}{l}\text { IEEE International Conf. on } \\
\text { Robotics and Automation }\end{array}$ & $\begin{array}{l}\text { International Conf. on Intelligent } \\
\text { User Interfaces Companion }\end{array}$ & Communications of the ACM \\
\hline $\begin{array}{l}\text { ACM/IEEE International Conf. on } \\
\text { Human-Robot Interaction }\end{array}$ & $\begin{array}{l}\text { International Conf. on Software } \\
\text { Eng. Companion }\end{array}$ & Interaction Studies (2 papers) \\
\hline $\begin{array}{l}\text { International Conf. on Intelligent } \\
\text { Systems and Computer Vision }\end{array}$ & Mensch und Computer Conference & $\begin{array}{l}\text { International Journal of Computer } \\
\text { Assisted Radiology and Surgery }\end{array}$ \\
\hline $\begin{array}{l}\text { IEEE-EMBS International Conf. on } \\
\text { Biomedical and Health Informatics }\end{array}$ & $\begin{array}{l}\text { ACM Conf. on Human-Computer } \\
\text { Interaction }\end{array}$ & $\begin{array}{l}\text { JMIR Rehabilitation and Assistive } \\
\text { Technologies }\end{array}$ \\
\hline $\begin{array}{l}\text { F1000Research } \\
\text { ReEE International Symposium on } \\
\text { Robot and Human Interactive Com. }\end{array}$ & $\begin{array}{l}\text { Journal of the American Medical } \\
\text { Informatics Association }\end{array}$ \\
\hline $\begin{array}{l}\text { International Conf. on Multimodal } \\
\text { Interaction }\end{array}$ & $\begin{array}{l}\text { Annual International Conf. of the } \\
\text { IEEE Eng. in Medicine and Biology } \\
\text { Society }\end{array}$ & Nature Medicine \\
\hline $\begin{array}{l}\text { ACM Conf. on Computer } \\
\text { Supported Cooperative Work \& } \\
\text { Social Computing }\end{array}$ & $\begin{array}{l}\text { World Congress on Medical and } \\
\text { Health Informatics }\end{array}$ & npj Digital Medicine \\
\hline $\begin{array}{l}\text { International Conf. Mixed Design } \\
\text { of Integrated Circuits and Systems }\end{array}$ & & $\begin{array}{l}\text { Procedia Computer Science (2 } \\
\text { papers) }\end{array}$ \\
\hline
\end{tabular}

\subsection{Distribution over diseases}

In terms of diseases, 15 papers examined human-AI collaboration in generic (not disease-specific) use cases, as seen in Figure 2. Of these, 4 papers focused on robotassisted surgery targeting at any disease, e.g., [51] [59]. Two papers developed coding systems which translated free-text physician notes into standards-based executable cohort definition queries [60] or diagnostic codes [55]. Two papers designed service robots for patients which provide interactive services, such question answering, chatting [42] and medication adherence [53]. Two papers developed solutions to assist nurses with various operations i.e., [34] [50]. One paper designed a collaborative environment to provide better rescue services for elderly [44]. Apart from the general-purpose studies, three papers targeted cancer diagnosis, i.e., skin cancer [57], prostate cancer [38], and breast cancer [58]. In addition, two papers focused on dementia, i.e., [36] [47]. The remaining 9 papers covered other individual diseases, as shown in Figure 2.

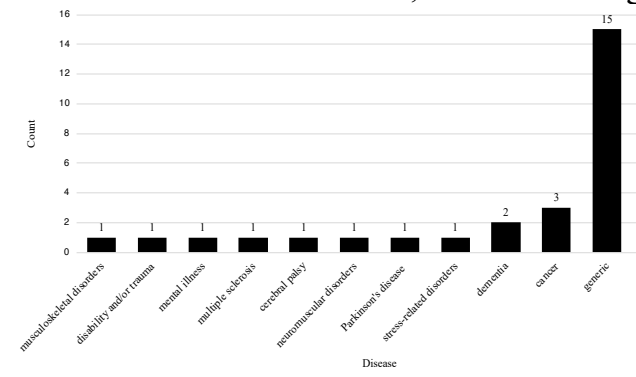

Figure 2. Number of papers across diseases

Although developing a generic solution for all diseases can seem efficient (as was seen in the majority of the 28 papers), it is important to understand whether considering characteristics of different diseases and their specialized care requirements in the AI system would further improve the quality of care. In this regard, it would be valuable to conduct more studies on humanAI collaboration, particularly for highly-prevalent and burdensome diseases.

\subsection{Distribution over clinical practices}

We further classified the papers based on targeted clinical practices. As Figure 3 shows, the most studied clinical practice is treatment (11), followed by robotassisted surgery (4) and diagnosis (4). For the 3 papers focusing on multiple practices, 2 papers developed multi-component AI systems for both diagnosis and treatment, i.e., [33] [35], while the remaining paper developed an assistive robot for helping nurses with various operations during patient care [50].

Apart from healthcare professional-AI collaboration and patient-AI collaboration, AI systems could also help clinical researchers through their ability to process massive volumes of free-text medical data [55] [60] and laboratory test results [41]. Two papers developed AI systems for disease prevention, i.e., dementia [47] and musculoskeletal disorders [40]. Among the clinical practices, human-AI collaboration in prognosis is relatively less studied, with only 1 paper that designed a predictive algorithm for multiple sclerosis [56]. Though there are currently relatively more studies on treatment practice, there is much potential for human-AI collaboration research in all clinical practice areas methods. As seen in Figure 4, most of the papers (23) adopted design and implementation as a research method. These papers focused on applying various techniques to design intelligent systems e.g., collaborative robots to achieve higher performance in healthcare as compared to humans acting alone without the support of AI. 


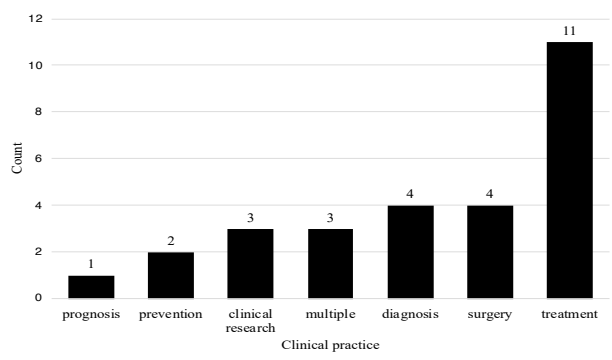

Figure 3. Number of papers across clinical practices

\subsection{Distribution over research methods}

In terms of distribution over research methods, we counted a paper multiple times if it adopted multiple methods. (Paper may appear in multiple categories.)

Twelve of these papers conducted experiments to evaluate the developed solutions. Of these, 4 papers performed field experiments with the AI systems evaluated in a natural setting [43] [52] [54] [58]. The other 8 papers conducted experiments in laboratory settings or in simulated environments [40] [48] [49] [46] [50] [51] [57] [59]. One common issue for both the field and lab experiments is the limited number of participants, ranging from 2 to 22 , except one study that hired 302 participants [57]. For example, Malik and colleagues developed an interactive robot for children with cerebral palsy but only evaluated this robot with 2 participants [52].

Three studies conducted qualitative interviews to collect users' feedback [38] [47] [54]. For example, Cai and colleagues [38] interviewed 21 pathologists before, during, and after being presented the AI prediction results for prostate cancer diagnosis, to learn the types of information that they desired from the AI assistant. Two papers performed case studies [45] [34], while one study each conducted a field study [47] and survey [54]. Overall, we see the potential for conducting more field studies and surveys to gain wider understanding, as well as case studies to obtain more in-depth insights.

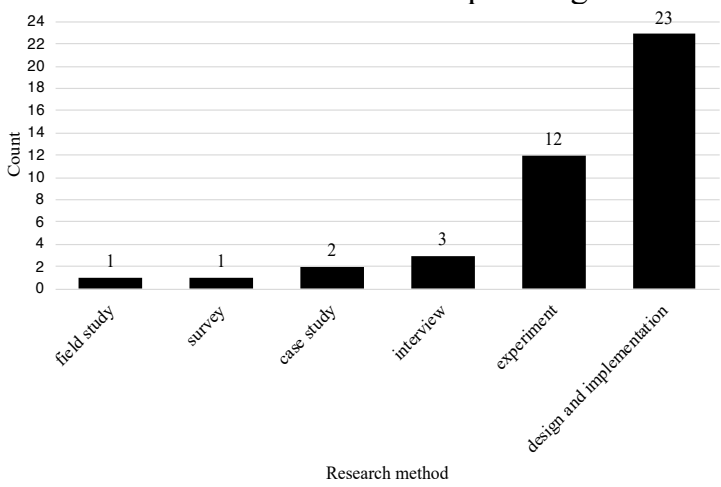

Figure 4. Number of sample papers across methods

\subsection{Themes of collaboration}

We identified the following themes from the 28 studies based on the stages (design and implementation of the AI artifact, its evaluation, adoption), the parties involved in collaboration, and finally the theories employed.

In terms of the design and implementation of $A I$ artifacts involved in collaboration, different AI techniques were employed, e.g., neural network models [38], random forest approach [56], and support vector machine and decision tree [36]. Also, different areas of AI were explored and integrated into the systems, e.g., computer vision [46], speech recognition [53], and natural language processing [55] [60]. Apart from the software components, some papers described the hardware design of the robots or AI medical devices in detail, e.g., a cable-driven robot [58].

With regards to evaluation, only 5 papers did not mention any evaluation of the collaborative system [35] [42] [44] [45] [53] and 1 paper described the proposed evaluation plan but did not actually conduct the evaluation [39]. Among the remaining papers, 8 papers [33] [36] [37] [41] [48] [51] [55] [56] only evaluated the performance of the AI system, without the users, and the metrics were statistically-calculated variables, i.e., true positives, true negatives, false positives, false negatives, sensitivity, specificity, accuracy, precision, recall, F1 score, and Area Under the ROC Curve (AUC) score.

Other papers evaluated the downstream outcomes from human-AI collaboration, e.g., task performance after collaboration [57] [58] [59], task completion time [46] [49] [57], and learning/training results after collaborating with interactive robots [43] [52]. In particular, 4 papers assessed usability and acceptability measures, such as difficulty to use/control [46] [50] [60], satisfaction [54], and safety [50].

Apart from 1 paper, which measured the willingness to use AI through a single item of usability [60], the remainder of the papers did not investigate the adoption of the collaborative AI in healthcare practice. Although it is suggested that collaborating with AI could be beneficial, for example in terms of performance improvement and reduction of task completion time, there are still open questions about their adoption and use. As indicated by Cai and colleagues [38], there are various considerations prior to adoption, for example, information on regulatory approval (e.g. FDA approval), peer-reviewed studies validating the artifact, impact on existing clinician workflows, impact on legal liability, and cost of purchase.

With regard to the parties involved in the collaborations, there are several types of stakeholders in the healthcare domain: healthcare professionals (e.g., physicians, surgeons, nurses, and assistants), patients, clinical researchers, and the AI systems. Fifteen papers 
addressed the collaboration between healthcare professionals and the AI system, e.g., predictive algorithms helping physicians with disease diagnosis [38] [57], and assistive robot during surgery [51]. Particularly, 1 study investigated the collaboration between a robot and the whole surgical team in the operation room [46]. Eight papers focused on patient-AI collaboration targeting different diseases, e.g., workrelated musculoskeletal disorders [40], mental illness [45] and Parkinson's disease [37]. Six papers developed solutions dedicated to vulnerable populations, e.g., children and the elderly who need specific care, with 3 for children [39] [43] [52] and 3 for the elderly [42] [44] [47]. Four papers investigated the collaboration among 3 entities, i.e., patients, AI artifacts, and human assistants/helpers [42] [47] [44] [54], while 2 papers looked at AI collaborations with researchers.

Finally, we found a lack of theory-driven papers in the review. Among all 28 papers, only 2 studies referred to theories to guide their research: 1) Hubbard and colleagues" work used the "serve and return" early childhood model and the constructionism theory of learning to design their interactive robot [43]; 2) Anya and colleagues' work used activity theory to guide the modeling of problem solving interactions during diagnosis and treatment [35]. The remaining papers examined human-AI collaboration without considering or investigating the theoretical mechanisms behind such collaboration.

Table 2. The syntheses of review results: findings, research gaps, and future directions

\begin{tabular}{|c|c|c|}
\hline Findings & Research gaps & Future directions \\
\hline $\begin{array}{l}\text { Research related to human-AI } \\
\text { collaboration in healthcare showed } \\
\text { an increase over time, but in terms } \\
\text { of absolute numbers, this is still } \\
\text { limited. }\end{array}$ & $\begin{array}{l}\text { With the challenges summarized in } \\
\text { the related work, there is still a } \\
\text { lack of related research in } \\
\text { healthcare as the absolute number } \\
\text { of studies are quite small. }\end{array}$ & $\begin{array}{l}\text { More studies should be conducted, } \\
\text { particularly on the topics mentioned } \\
\text { below. }\end{array}$ \\
\hline $\begin{array}{l}\text { Most of the peer-reviewed papers } \\
\text { are published in conferences across } \\
\text { multiple fields. }\end{array}$ & $\begin{array}{l}\text { The number of IS outlets covered } \\
\text { is limited. }\end{array}$ & $\begin{array}{l}\text { IS outlets should be more receptive } \\
\text { to this topic and encourage relevant } \\
\text { submissions. }\end{array}$ \\
\hline $\begin{array}{l}\text { More than half of the reviewed } \\
\text { papers examined human-AI } \\
\text { collaboration in generic use cases, } \\
\text { followed by cancer and dementia. }\end{array}$ & $\begin{array}{l}\text { We identified the uneven } \\
\text { distribution of past research in } \\
\text { terms of the disease context } \\
\text { studied. }\end{array}$ & $\begin{array}{l}\text { More studies should be conducted on } \\
\text { human-AI collaboration particularly } \\
\text { for highly-prevalent and burdensome } \\
\text { diseases. Specific characterizes of } \\
\text { different disease types could be } \\
\text { considered. }\end{array}$ \\
\hline $\begin{array}{l}\text { Most of the reviewed papers } \\
\text { focused on treatment, followed by } \\
\text { surgery and diagnosis. }\end{array}$ & $\begin{array}{l}\text { We identified an uneven } \\
\text { distribution in terms of clinical } \\
\text { practices. }\end{array}$ & $\begin{array}{l}\text { Future research could examine } \\
\text { human-AI collaboration in less- } \\
\text { studied clinical practices, such as } \\
\text { disease prevention. }\end{array}$ \\
\hline $\begin{array}{l}\text { Most of the papers adopted design } \\
\text { and implementation as a research } \\
\text { method. }\end{array}$ & $\begin{array}{l}\text { Compared to design and } \\
\text { implementation and experiment, } \\
\text { other research methods (e.g., } \\
\text { surveys, field studies) are less } \\
\text { common. }\end{array}$ & $\begin{array}{l}\text { It is valuable to conduct more field } \\
\text { studies and surveys to gain wider } \\
\text { understanding and more in-depth } \\
\text { insights. }\end{array}$ \\
\hline $\begin{array}{l}\text { Research stages: } \\
\text { 1) for design and implementation, } \\
\text { different AI techniques were } \\
\text { employed, and various areas of AI } \\
\text { were explored and integrated into } \\
\text { the systems } \\
\text { 2) for evaluation, some papers did } \\
\text { not mention evaluation, while } \\
\text { others papers only evaluated the } \\
\text { performance of the AI systems } \\
\text { 3) for adoption, only one paper } \\
\text { measured the willingness to use AI } \\
\text { through usability perspective. }\end{array}$ & $\begin{array}{l}\text { 1) The reviewed studies focused on } \\
\text { very few downstream outcomes } \\
\text { from human-AI collaboration. } \\
\text { 2) The absolute number of papers } \\
\text { focused on adoption is quite small. }\end{array}$ & $\begin{array}{l}\text { 1) More behavioral outcomes and } \\
\text { social issues should be examined and } \\
\text { evaluated. } \\
\text { 2) Instead of single task and short- } \\
\text { term collaborative outcomes, } \\
\text { multiple tasks, evolving and } \\
\text { interactive collaboration processes, } \\
\text { and long-term impacts should be } \\
\text { examined. } \\
\text { 3) The organizational outcomes } \\
\text { should be considered. } \\
\text { 4) More studies are needed to focus } \\
\text { on adoption. }\end{array}$ \\
\hline
\end{tabular}




\begin{tabular}{|l|l|l|}
\hline $\begin{array}{l}\text { There are four types of } \\
\text { stakeholders in the healthcare } \\
\text { domain: healthcare professionals, } \\
\text { patients, clinical researchers, and } \\
\text { the AI systems. }\end{array}$ & $\begin{array}{l}\text { Most of the papers focused on the } \\
\text { collaboration between only two } \\
\text { parties, especially between } \\
\text { healthcare professionals and AI } \\
\text { systems. There are relatively fewer } \\
\text { studies on patient-AI and } \\
\text { researcher-AI collaboration. }\end{array}$ & $\begin{array}{l}\text { More studies could be conducted to } \\
\text { investigate collaboration between } \\
\text { three or more parties which are not } \\
\text { uncommon contexts, e.g., } \\
\text { collaboration among physicians, } \\
\text { patients, and the AI. }\end{array}$ \\
\hline $\begin{array}{l}\text { Only two studies referred to } \\
\text { theories to guide their research. }\end{array}$ & $\begin{array}{l}\text { More theory-driven research is } \\
\text { needed to understand the } \\
\text { mechanisms for human to } \\
\text { collaborate effectively with AI } \\
\text { systems. }\end{array}$ & $\begin{array}{l}\text { 1) Future research could investigate } \\
\text { the generalizability of inter-personal } \\
\text { collaboration theories to human-AI } \\
\text { collaboration. } \\
\text { 2) More research can focus on the } \\
\text { complementary expertise of human } \\
\text { professionals and the AI system. } \\
\text { 3) Emotional factors and related } \\
\text { theories could be salient in human-AI } \\
\text { collaboration, which may be } \\
\text { effective at guiding the design of AI } \\
\text { artifacts. }\end{array}$ \\
\hline
\end{tabular}

\section{Future directions}

Our findings show an increase in publications about human-AI collaboration in healthcare over time (see Figure 1). As we summarized in the Related Work section, there are many challenges that IS researchers can provide meaningful inputs on, and there is a lack of existing research (as indicated by our review) especially in IS outlets. This can also be done in conjunction with collaborators from related disciplines, as research in this area often requires multi-disciplinary expertise.

Second, our findings reveal the uneven distribution of past research in terms of diseases (see Figure 2) and clinical practices (see Figure 3). Although generalizable AI dealing with multiple diseases using one solution is cost-effective, considering specific characteristics of different disease types may contribute to achieving more effective collaboration. Future research could examine human-AI collaboration in less-studied clinical practices, such as prognosis and prevention; though diagnosis and treatment are the major practices [8], a focus on disease prevention is increasingly being advocated. In addition, the characteristics of different population segments could also be investigated for promoting human-AI collaboration in healthcare. For example, children are considered non-collaborative patients, and less than half of young children are testable with current screening tools [22].

Third, we found that most of the papers adopted design and implementation as their research method. Instead of solely focusing on the development of the AI artifact, it is valuable to conduct more field studies and surveys to obtain deeper understanding in practice.

Fourth, as indicated by our results, only half of the papers (14) evaluated human-AI collaborative outcomes in healthcare, as well as the usability and acceptability of the collaborative technologies. The remaining 14 papers either did not mention evaluation or only evaluated the performance of the AI systems working alone. IS researchers could contribute towards investigating behavioral outcomes and social issues pertaining to ethics, interpretability, accountability, autonomy/control, conflict resolution, trust and cohesion in such collaborations. Furthermore, current studies mainly investigated human-AI collaboration in a single task. However, as we defined in the introduction, collaboration is an evolving, interactive process. Thus, the interactivity of AI systems should be examined for supporting collaboration. Apart from short-term collaborative outcomes, future research should investigate the long-term impacts of human-AI collaboration in healthcare, such as how collaboration might change healthcare professionals' competences, job roles, and career development in future.

Additionally, it is often unclear whether humans and AI are optimizing for the same metrics. While algorithms are optimized for a specific metric, healthcare professionals might consider other factors, e.g., ethical considerations in the diagnosis of mental illness. As Cai and colleagues' study indicated [38], it is also important to examine the AI's medical point-ofview, e.g., its source of ground truth and diagnostic tendency. It may be useful to design methods for AI to take physicians' recommendations at runtime and evaluate whether this could improve AI performance.

Further, by considering an organizational perspective, IS researchers could examine how healthcare organizations can benefit from the power of human-AI collaboration, as well as how they should adapt their workflow integration, reconfiguration, and 
coordination for this purpose. As workflows and roles would change, future research could investigate how organizations can facilitate such change by training people in the required collaboration competences. At the societal level, more widespread adoption of human-AI collaboration would necessitate a rethinking of medical education programs and how they should be updated.

With respect to adoption, more studies are needed. From healthcare professionals' perspective, there is a fundamental question about the professional's perceptions of the $\mathrm{AI}$ : when collaborating with $\mathrm{AI}$, do they perceive $\mathrm{AI}$ as a teammate, or do they treat the $\mathrm{AI}$ as a tool? For example, it is common for physicians to seek a second opinion from peers. Ideally, it should not make a difference whether the peer is another human physician or an AI system. However, it seems likely that when a physician perceives an AI system as a tool, instead of collaborative decision-making we would see an authoritative mode where the physician may override the system recommendations, e.g., of stat doses [32]. In the worst case, even though the AI might be welldeveloped and trained, its intelligence would not be utilized in practice. To understand people's psychological perceptions of a machine partner, Wynne and Lyons [30] proposed the concept of autonomous agent teammate-likeness (ATT) as the extent to which a human perceives and identifies an autonomous, intelligent agent partner as a highly altruistic, benevolent, interdependent, emotive, and communicative teammate. It is worth investigating this construct- or applying other human-agent theories for human-AI collaboration in the healthcare context.

Fifth, our results show that so far there could be four types of stakeholders in human-AI collaboration in healthcare. Most of the papers investigated the pairwise collaboration between the AI systems and the three human parties, particularly the collaboration between the healthcare professionals and AI. There are opportunities for future research to study collaboration between three and more parties, e.g., the integrated AIbased diagnosis platform for patients and physicians.

Last, more theory-driven research is needed to understand the mechanisms for human actors to collaborate effectively with AI systems, the impact brought on by such collaborations, and the factors leading to successful collaborations. Future research could also investigate the generalizability of interpersonal collaboration theories to human-AI collaboration. Such theory-driven research could complement the current, major focus on the design and implementation of collaborative AI-systems (see Figure 4). Even for the design of these systems, it would be important to consider the complementarity of expertise between the AI and the human, rather than only on what the AI can do. Furthermore, emotional factors may be salient in effective collaboration [5], e.g., reactions to a robot co-worker. Research could be conducted to investigate the effects of these factors in human-AI collaborations through affective computing.

In conclusion, this paper provides a timely review of the literature on human-AI collaboration in healthcare, identifies gaps in our understanding, and outlines future directions for IS research (summarized in Table 2).

\section{Limitations}

This review paper has several limitations that should be considered and extended in the future work. First, we can add more keywords and synonyms (e.g., "teamwork") into the search query to increase our search recall. Second, we could include more databases across various disciplines. Third, we could use more sophisticated information retrieval tools. For example, we could use topic modelling tools to identify potentially relevant groups of words, allowing us to iterate and add more to our search filters.

\section{References}

[1] Armstrong, D., "Clinical autonomy, individual and collective: the problem of changing doctors' behaviour", Social Science \& Medicine (55:10), 2002, pp.1771-1777.

[2] Allied Market Research, "Autonomous Vehicle Market Expected to Reach \$54.23 Billion, by 2026", https://www.alliedmarketresearch.com/press/release/aut onomous-vehicle-market.html, 2019.

[3] Bedwell, W.L., Wildman, J.L., DiazGranados, D., Salazar, M., Kramer, W.S. and Salas, E., "Collaboration at work: An integrative multilevel conceptualization", Human Resource Management Review (22:2), 2012, pp. 128145.

[4] Brown, S.A., Dennis, A.R., and Venkatesh, V., "Predicting collaboration technology use: Integrating technology adoption and collaboration research", Journal of Management Information Systems (27:2), 2010, pp. 954.

[5] Cox, J.D., "Emotional intelligence and its role in collaboration", Proceedings of ASBBS (18:1), 2011, pp. 435-445.

[6] Deloitte Insights, "Superteams -putting AI in the group", https://www2.deloitte.com/us/en/insights/focus/humancapital-trends/2020/human-ai-collaboration.html, 2020.

[7] Digital Trends, "Revisiting the rise of A.I.: How far has artificial intelligence come since 2010?", https://www.digitaltrends.com/cool-tech/biggest-aiadvances-of-the-2010s/, 2019.

[8] Gill, T.M., "The central role of prognosis in clinical decision making", The Journal of the American Medical Association (307:2), 2012, pp. 199-200.

[9] Hao, K., "We analyzed 16,625 papers to figure out where AI is headed next", MIT Technology Review, 2019.

[10] Health IT Analytics, "Arguing the Pros and Cons of Artificial Intelligence in Healthcare", https://healthitanalytics.com/news/arguing-the-pros-andcons-of-artificial-intelligence-in-healthcare, 2018. 
[11] Jain, S. and Argall, B., "Probabilistic human intent recognition for shared autonomy in assistive robotics", ACM Transactions on Human-Robot Interaction (9:1), 2019, pp. 1-23.

[12] Khan, K.S., Kunz, R., Kleijnen, J. and Antes, G., "Five steps to conducting a systematic review", Journal of the Royal Society of Medicine (96:3), 2003, pp. 118-121.

[13] Lai, Y., Sukhwal, P.C. and Kankanhalli, A., "Explanations in Clinical DSS: A Review and Research Agenda", Pacific Asia Conference on Information Systems, 2020, p. 169.

[14] Lindgren, H, "Towards personalized decision support in the dementia domain based on clinical practice guidelines", User Modeling and User-Adapted Interaction (21:4-5), 2011, pp. 377-406.

[15] McKinsey \& Company, "The big-data revolution in US health care: Accelerating value and innovation", https://www.mckinsey.com/industries/healthcaresystems-and-services/our-insights/the-big-datarevolution-in-us-health-care, 2013.

[16] Miner, A.S., Shah, N., Bullock, K.D., Arnow, B.A., Bailenson, J. and Hancock, J., "Key considerations for incorporating conversational AI in psychotherapy", Frontiers in Psychiatry (10), 2019.

[17] Olive AI, "Half of hospital decision makers plan to invest in AI by 2021", https://oliveai.com/blog/hospitaldecision-makers-plan-to-invest-in-ai/, 2019.

[18] Pacis, D.M.M., Subido Jr, E.D. and Bugtai, N.T., "Trends in telemedicine utilizing artificial intelligence", AIP Conference Proceedings (1933:1), 2018, p. 040009.

[19] Park, S.Y., Kuo, P.Y., Barbarin, A., Kaziunas, E., Chow, A., Singh, K., Wilcox, L. and Lasecki, W.S., "Identifying Challenges and Opportunities in Human-AI Collaboration in Healthcare," Conference Companion Publication of the 2019 on Computer Supported Cooperative Work and Social Computing, 2019, pp. 506510.

[20] PR Newswire, "New Virtual Caregiver Technology May Reduce Long-term Care Costs by up to 92 Percent by 2050", https://www.prnewswire.com/newsreleases/new-virtual-caregiver-technology-may-reducelong-term-care-costs-by-up-to-92-percent-by-2050300784454.html, 2019.

[21] Price II, W.N., "Artificial Intelligence in Health Care: Applications and Legal Implications", https://www.americanbar.org/groups/science_technolog y/publications/scitech_lawyer/2017/fall/artificialintelligence-health-care/,2017.

[22] Pueyo, V., Pérez-Roche, T., Prieto, E., Castillo, O., Gonzalez, I., Alejandre, A., Pan, X., Fanlo-Zarazaga, A., Pinilla, J., Echevarria, J.I. and Gutierrez, D., "Development of a system based on artificial intelligence to identify visual problems in children: study protocol of the TrackAI project", BMJ Open 10(2), 2020.

[23] Seeber, I., Bittner, E., Briggs, R.O., de Vreede, T., De Vreede, G.J., Elkins, A., Maier, R., Merz, A.B., OesteReiß, S., Randrup, N. and Schwabe, G., "Machines as teammates: A research agenda on AI in team collaboration", Information \& management (57:2), 2020, p. 103174.
[24] Souissi, S.B., Abed, M., El Hiki, L., Fortemps, P. and Pirlot, M, "PARS, a system combining semantic technologies with multiple criteria decision aiding for supporting antibiotic prescriptions", Journal of Biomedical Informatics (99), 2019, p. 103304.

[25] The Guardian, "Doctors at breaking point in underfunded NHS",

https://www.theguardian.com/society/2019/apr/01/docto rs-at-breaking-point-in-underfunded-nhs, 2019.

[26] Vedula, S.S. and Hager, G.D., "Surgical data science: The new knowledge domain", Innovative Surgical Sciences (2:3), 2017, pp. 109-121.

[27] Wang, P., "What Do You Mean by “AI"'?", Artificial General Intelligence (171), 2008, pp. 362-373.

[28] Wilson, H. J., and Daugherty, P. R. "Collaborative intelligence: Humans and AI are joining forces", Harvard Business Review, 96(4), 2018, pp. 114-123.

[29] World Health Organization, "World Health Statistics 2019: Monitoring health for the SDGs", https://www.who.int/gho/publications/world_health_stat istics/2019/en/, 2019.

[30] Wynne, K.T. and Lyons, J.B., "An integrative model of autonomous agent teammate-likeness", Theoretical Issues in Ergonomics Science (19:3), 2018, pp. 353-374.

[31] Xie, Y., Bodala, I.P., Ong, D.C., Hsu, D. and Soh, H., "Robot capability and intention in trust-based decisions across tasks", The 14th ACM/IEEE International Conference on Human-Robot Interaction, 2019, pp. 3947.

[32] Yang, Z., Ng, B. Y., Kankanhalli, A., and Yip, J. W. L., "Workarounds in the use of IS in healthcare: A case study of an electronic medication administration system", International Journal of Human-Computer Studies, 2012, (70:1), pp. 43-65.

\section{Appendix - the 28 peer-reviewed papers $(2$}

* papers are treated as 1 study as they belong to 1 project)

[33] Ahmed, M.U., Begum, S. and Funk, P., "A hybrid casebased system in clinical diagnosis and treatment", Proceedings of 2012 IEEE-EMBS International Conference on Biomedical and Health Informatics, 2012, pp. 699-704.

[34] Ahn, H.S., Lee, M.H. and MacDonald, B.A., "Healthcare robot systems for a hospital environment: CareBot and ReceptionBot", The 24th IEEE International Symposium on Robot and Human Interactive Communication, 2015, pp. 571-576.

[35] Anya, O., Tawfik, H. and Nagar, A., "Cross-boundary knowledge-based decision support in e-health", 2011 International Conference on Innovations in Information Technology, 2011, pp. 150-155.

[36] Bang, S., Son, S., Roh, H., Lee, J., Bae, S., Lee, K., Hong, C. and Shin, H., "Quad-phased data mining modeling for dementia diagnosis", BMC Medical Informatics and Decision Making 17(1), 2017, p. 60.

[37] Cabestany, J., López, C.P., Sama, A., Moreno, J.M., Bayes, A. and Rodriguez-Molinero, A., "REMPARK: When AI and technology meet Parkinson Disease assessment", Proceedings of the 20th International Conference Mixed Design of Integrated Circuits and Systems, 2013, pp. 562-567. 
[38] Cai, C.J., Winter, S., Steiner, D., Wilcox, L. and Terry, M., "" Hello AI": Uncovering the Onboarding Needs of Medical Practitioners for Human-AI Collaborative Decision-Making", Proceedings of the ACM on HumanComputer Interaction (3:CSCW), 2019, pp. 1-24.

[39] Calderita, L.V., Manso, L.J., Bustos, P., Suárez-Mejías, C., Fernández, F. and Bandera, A., "THERAPIST: towards an autonomous socially interactive robot for motor and neurorehabilitation therapies for children", JMIR Rehabilitation and Assistive Technologies (1:1), 2014, p. e1.

[40] El Makrini, I., Merckaert, K., De Winter, J., Lefeber, D. and Vanderborght, B., "Task allocation for improved ergonomics in Human-Robot Collaborative Assembly", Interaction Studies (20:1), 2019, pp. 102-133.

[41] Fillmore, N., Do, N.V., Brophy, M. and Zimolzak, A.J., "Interactive Machine Learning for Laboratory Data Integration", World Congress on Medical and Health Informatics, 2019, pp. 133-137.

[42] Golchinfar, D., Vaziri, D.D., Schreiber, D. and Stevens, G., "Assisting Service Robots on their Journey to Become Autonomous Agents: From Apprentice to Master by Participatory Observation", Proceedings of Mensch und Computer, 2019, pp. 889-891.

[43] Hubbard, L.J., Erkocevic, B., Cassady, D., Cheng, C.H., Chamorro, A. and Yeh, T., "MindScribe: Toward Intelligently Augmented Interactions in Highly Variable Early Childhood Environments", Proceedings of the 23rd International Conference on Intelligent User Interfaces Companion, 2018, pp. 1-2.

[44] Humayoun, S.R., Avtandilov, A., Mehdi, S.A., Ebert, A. and Berns, K., "Live-Feedback Supported Collaborative Environment for Emergency Scenarios", In Proceedings of the 18th ACM Conference Companion on Computer Supported Cooperative Work \& Social Computing, 2015, pp. 147-150.

[45] Hussain, N., Wang, H.H. and Buckingham, C., "Policy based generic autonomic adapter for a context-aware social-collaborative system", International Conference on Intelligent Systems and Computer Vision 2018, 2018, pp. 1-9.

[46] Jacob, M.G., Li, Y.T., Akingba, G.A. and Wachs, J.P., "Collaboration with a robotic scrub nurse", Communications of the ACM (56:5), 2013, pp. 68-75.

[47] Jeon, C., Shin, H., Kim, S. and Jeong, H., "Talking over the robot: A field study of strained collaboration in a dementia-prevention robot class", Interaction Studies (21:1), 2020, pp.85-110.

[48] Jiang, Y., Liu, H., Kong, H., Wang, R., Hosseini, M., Sun, J. and Sha, L., "Use runtime verification to improve the quality of medical care practice", Proceedings of the 38th International Conference on Software Engineering Companion, 2016, pp. 112-121.

[49] Kaplan, K.E., Nichols, K.A. and Okamura, A.M., "Toward human-robot collaboration in surgery: performance assessment of human and robotic agents in an inclusion segmentation task", 2016 IEEE International Conference on Robotics and Automation, 2016, pp. 723729.
[50] Lee, W., Park, J. and Park, C.H., “Acceptability of Teleassistive Robotic Nurse for Human-Robot Collaboration in Medical Environment", Companion of the 2018 ACM/IEEE International Conference on Human-Robot Interaction, 2018, pp. 171-172.

* [51] Levy, A., Msellati, J. and De Muer, A., "An Innovative Approach to Safe Surgical Suturing Part I: Experimental Setup and Tests Protocol", In Proceedings of the 2019 9th International Conference on Biomedical Engineering and Technology, 2019, pp. 280-284.

* [51] Levy, A., Msellati, J. and De Muer, A., “An Innovative Approach to Safe Surgical Suturing Part II: Data Machine Learning Predictive Analysis", Proceedings of the 2019 9th International Conference on Biomedical Engineering and Technology, 2019, pp. 285-288.

[52] Malik, N.A., Yussof, H., Hanapiah, F.A., Rahman, R.A.A. and Basri, H.H., "Human-robot interaction for children with cerebral palsy: Reflection and suggestion for interactive scenario design", Procedia Computer Science (76), 2015, pp. 388-393.

[53] Mangipudi, V. and Tumuluri, R., "Context-Aware Multimodal Robotic Health Assistant", Proceedings of the 16th International Conference on Multimodal Interaction, 2014, pp. 76-77.

[54] Miyachi, T., Iga, S. and Furuhata, T., "Human robot communication with facilitators for care robot innovation", Procedia Computer Science (112), 2017, pp. 1254-1262.

[55] Nie, A., Zehnder, A., Page, R.L., Zhang, Y., Pineda, A.L., Rivas, M.A., Bustamante, C.D. and Zou, J., "DeepTag: inferring diagnoses from veterinary clinical notes", npj Digital Medicine (1:1), 2018, pp.1-8.

[56] Tacchella, A., Romano, S., Ferraldeschi, M., Salvetti, M., Zaccaria, A., Crisanti, A. and Grassi, F., "Collaboration between a human group and artificial intelligence can improve prediction of multiple sclerosis course: a proofof-principle study", F1000Research (6), 2017.

[57] Tschandl, P., Rinner, C., Apalla, Z., Argenziano, G., Codella, N., Halpern, A., Janda, M., Lallas, A., Longo, C., Malvehy, J. and Paoli, J., "Human-computer collaboration for skin cancer recognition", Nature Medicine, 2020, pp.1-6.

[58] Vitrani, M.A., Marx, A., Iordache, R., Muller, S. and Morel, G., "Robot guidance of an ultrasound probe toward a $3 \mathrm{D}$ region of interest detected through X-ray mammography", International Journal of Computer Assisted Radiology and Surgery (10:12), 2015, pp. 18931903.

[59] Xiong, J., Xia, Z. and Gan, Y., "Human-robot collaborated path planning for bevel-tip needle steering in simulated human environment", The 38th Annual International Conference of the IEEE Engineering in Medicine and Biology Society, 2016, pp. 5672-5675.

[60] Yuan, C., Ryan, P.B., Ta, C., Guo, Y., Li, Z., Hardin, J., Makadia, R., Jin, P., Shang, N., Kang, T. and Weng, C., "Criteria2Query: a natural language interface to clinical databases for cohort definition", Journal of the American Medical Informatics Association (26:4), 2019, pp. 29430 